\title{
Guidance to Pre-tokeniztion for SacreBLEU: Meta-Evaluation in Korean
}

\author{
Ahrii Kim ${ }^{1}$ and Jinhyun Kim ${ }^{2}$ \\ Kakao Enterprise \\ 235 Pangyoeok-ro Bundang-gu Seongnam-si, \\ Gyeonggi-do, Republic of Korea \\ ria.i, rob.kekakaoenterprise.com
}

\begin{abstract}
SacreBLEU, by incorporating a text normalizing step in the pipeline, has been well-received as an automatic evaluation metric in recent years. With agglutinative languages such as Korean, however, the metric cannot provide a conceivable result without the help of customized pre-tokenization.

In this regard, this paper endeavors to examine the influence of diversified pre-tokenization schemes -word, morpheme, character, and subword- on the aforementioned metric by performing a meta-evaluation with manuallyconstructed into-Korean human evaluation data.
\end{abstract}

Our empirical study demonstrates that the correlation of SacreBLEU (to human judgment) fluctuates consistently by the token type. The reliability of the metric even deteriorates due to some tokenization, and $\mathrm{MeCab}$ is not an exception. Guiding through the proper usage of tokenizer for each metric, we stress the significance of a character level and the insignificance of a Jamo level in MT evaluation.

Link to our code is available at http:// github.com/ko_sacrebleu

\section{Introduction}

For almost two decades, BLEU (Papineni et al., 2002) has played a vital role in Machine Translation (MT) evaluation as an all-time favored automatic metric, whether we actually "like" it or not. Marie et al. (2021) statistically backed up such a trend, reporting that in the past decade (2010-2020), about $98.8 \%$ of research papers submitted in ACL under the title of MT regarded BLEU as a prime evaluation metric. Although we kept getting stern warnings against its use (Tan et al. 2015; CallisonBurch et al. 2006), 89\% of new cutting-edge metrics that exhibited better correlation with human judgment (than other metrics including BLEU) were never deployed in actual researches to date
(Marie et al., 2021). The research community opted for stabilizing it instead of exploring new ones, and the best alternative seemed to be SacreBLEU (Post, 2018).

The biggest strength of SacreBLEU was that it reduced the influence of pre-processing scheme on the score computation that could have otherwise fluctuated upon any minor changes such as a type of tokenizers, a split of compound nouns, use of unknown tokens for rare words or casing (Post, 2018). By embracing the text normalizing step in the architecture, SacreBLEU could provide more trustworthy evaluation scores.

While it was gaining weight in the literature, the trust issue was still prominent in agglutinate languages such as Korean. Languages of such typology by design required language-specific tokenization to consider semantic features, which was absent from the pipeline of SacreBLEU. The rule of thumb was to use MeCab-ko ${ }^{1}$ beforehand as directed in Workshop on Asian Translation (Nakazawa et al., 2020), but its correlation to human judgment in MT evaluation was not officially confirmed.

In the context where Korean is not capable of taking advantage of SacreBLEU's protective layer, we shed light on the influence of a varied pre-tokenization pipeline on the given automatic metric that features three lexical-similarity-based types: BLEU, TER (Snover et al., 2006), and ChrF (Popović, 2015). At the same time, we share empirical lessons for SacreBLEU when applying it in the Korean language in MT evaluation, some of which are as follows.

At the segment level:

1. The pre-tokenization enhances the credibility of BLEU and TER in almost all cases.

2. MeCab is beneficial to BLEU and TER, but

\footnotetext{
${ }^{1}$ https://bitbucket.org/eunjeon/ mecab-ko
} 


\begin{tabular}{|c|c|c|c|c|c|}
\hline Level & Denomination $\beta$ & Particle & Ending & Affix & Example \\
\hline \multirow{3}{*}{ Word } & Eojeol & $\mathrm{X}$ & $\mathrm{X}$ & $\mathrm{X}$ & 혜미가, 동화를, 읽었다 \\
\hline & Word & $\mathrm{O}$ & $\mathrm{X}$ & $\mathrm{X}$ & 혜미, -가, 동화, -를, 읽었다 \\
\hline & Word & $\mathrm{O}$ & $\mathrm{O}$ & $\mathrm{X}$ & 혜미, -가, 동화, -를, 읽, -었다 \\
\hline Morpheme & Morpheme & $\mathrm{O}$ & $\mathrm{O}$ & $\mathrm{O}$ & 혜미, -가, 동화, -를, 읽, -었, -다 \\
\hline Syllable & Eumjeol & - & - & - & 혜, -미, -가, 동, -화, -를, 읽, -었, -다 \\
\hline Consonant \& Vowel & Jamo & - & - & - & 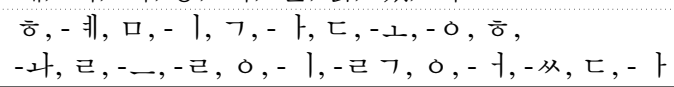 \\
\hline
\end{tabular}

Table 1: Definition of a Korean word (Nam et al., 2019) in comparison to other meta-levels.

when it comes to ChrF, there is a good chance that it damages its reliability.

3. The influence of the subword level is insignificant. In the worst case, the segmentation at this level could even degrade the credibility of ChrF and TER.

4. The segmentation at character level could substitute other token units, especially in BLEU and $\mathrm{ChrF}$.

At the corpus level:

1. The metric score can be inflated up to twice when tokenized.

2. The superiority among systems measured by SacreBLEU does not comply with human judgment. The mean value of the segmentlevel scores can be a safe option at this moment.

\section{Background}

\subsection{Word Segmentation}

A single distinct meaningful element of speech or writing, $[. .$.$] and typically$ shown with a space on either side when written or printed. ${ }^{2}$

The general definition of word, as shown above, conjectures that it is separated by space. Such assumption, however, is arguable in Korean, whose word does not always come with a space on either side.

As displayed in Table 1, there are three approaches in defining a word: comprehensive, compromising, and analytic. They diverge on the endowment of qualification as an independent element to three components - post-positional particle, ending, and affix (Nam et al., 2019). Following the

\footnotetext{
${ }^{2}$ https:// www.oxfordlearnersdictionaries. com
}

comprehensive standpoint, what is typically understood as a word in Western languages is equivalent to Eojeol in Korean. The compromising perspective sees that endings and affixes are not independent while the analytic approach recognizes the self-reliance of the endings. That much active discussion is possible with the morpheme boundary, which makes the Korean word decomposition complex.

In this paper, we describe two peculiar aspects of the Korean letter (or Hangeul). In the first place, a character has a sub-layer. The word read, for instance, is composed of four characters: r-e-a-d. The equivalent Korean word 읽 in Table 1 is also a character, but at the same time it is a combination of two consonants ( $O$, 리 ) and one vowel ( I). We call this sub-layer Jamo ( o - l- 27 ).

Secondly, Jamo are position-wise; they are situated in a fixed position of Choseong (initial, o ), Jungseong (middle, I), and Jongseong (final, 가), respectively. Some affixes or morphemes take the form of Jongseong, making a diversified token scenario between the morpheme and Jamo level possible. The elaborate example is given in Table 2.

\subsection{Token Level}

We define four meta-levels of segmentation for our experiment: word, morpheme, character, and subword. As discussed in Section 2.1, particle (or Josa), ending, and affix are the fork of a road to the classification of the tokens.

- Word: We adopt the comprehensive perspective. Hence, this token level is conceptually equivalent to Eojeol, which does not consider particles, ending or affixes as an independent element.

- Morpheme: This token level considers particles, endings, and affixes independent. The level of segmentation varies from tokenizer to tokenizer. 


\begin{tabular}{|c|c|c|c|c|c|c|c|c|c|c|c|c|c|c|c|c|c|c|c|c|c|c|c|c|c|c|c|c|c|c|c|c|c|c|}
\hline \multirow[t]{2}{*}{ Word } & & \multicolumn{2}{|c|}{ 모델 } & \multicolumn{2}{|c|}{ 레옹 } & \multicolumn{3}{|c|}{ 데임 } & \multirow[t]{2}{*}{ 은 } & \multicolumn{2}{|c|}{ 아직 } & \multirow[t]{2}{*}{ 그 } & \multicolumn{3}{|c|}{ 누구도 } & \multicolumn{3}{|c|}{ 시도한 } & \multirow{2}{*}{\multicolumn{2}{|c|}{ 적 }} & \multicolumn{2}{|l|}{ 없는 } & \multicolumn{3}{|c|}{ 방식으로 } & & \multicolumn{4}{|c|}{ 캣워크를 } & \multicolumn{4}{|c|}{ 활보했다 } \\
\hline & & & & 레 & 옹 & 데 & 임 & & & & & & 누구 & 도 & & 시도 & & 하 & L & & 없 & 는 & 방식 & & 으로 & & 캣워크 & 를 & & & 활보 & 했다 & & \\
\hline \multirow{4}{*}{\multicolumn{2}{|c|}{ Morpheme }} & & & & & 데 & 이 & 口 & & & & & 누 & 구도 & & & & & & & & & & & & & 캣 & 워크 & 를 & & 활보 & 犃 & 다 & \\
\hline & & & & & & & & & & & & & & & & & & & & & & & & & & & 캐 & 엇 & 워크 & 를 & 활 & 보 & 했 & 다 \\
\hline & & & & & & & & & & & & & & & & & & & & & & & & & & & & & & & 활보 & 하 & 었 & 다 \\
\hline & & & & & & & & & & & & & & & & & & & & & & & & & & & & & & & 활보 & 하 & 았 & 다 \\
\hline Character & & 모 & 델 & 레 & 옹 & 데 & 임 & & 은 & 아 & 직 & 그 & 누 & 구 & 도 & 시 & 도 & 한 & & 적 & 없 & 는 & 방 & 식 & 으 & 로 & 캣 & 워 & 크 & 를 & 활 & 보 & 腤 & 다 \\
\hline \multirow{3}{*}{ Subword } & choseong & ㅁ & ᄃ & ᄅ & o & ᄃ & o & & o & o & x & 7 & ᄂ & 7 & ᄃ & 人 & ᄃ & $\bar{\partial}$ & & x & o & L & ㅂ & 人 & o & ᄅ & $\Rightarrow$ & o & $\Rightarrow$ & ᄅ & $\bar{\sigma}$ & ㅂ & $\bar{\sigma}$ & ᄃ \\
\hline & jungseong & $\perp$ & 게 & ㄱ & $\perp$ & ㅔ & 1 & & - & t & 1 & - & $T$ & $T$ & $\perp$ & 1 & $\perp$ & t & & 1 & 1 & - & r & 1 & - & $\perp$ & H & 겨 & - & - & 과 & $\perp$ & H & t \\
\hline & $\overline{\text { jongseong }}$ & & ᄅe & & H & & ㅁ & & ᄂ & & 7 & & & & & & & ᄂ & & 7 & 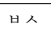 & ᄂ & o & 7 & & & 人 & & & ᄅ & ᄅ & & 从 & \\
\hline
\end{tabular}

Table 2: All possible tokenization schemes with the tokenizers applied in this study.

- Character: This token level is morphologically similar to but semantically different from Eumjeol or syllable. The segmentation strategy of this level does not require a tokenizer.

- Subword: This token level is the smallest token unit in Korean. A character is decomposed into Jamo, and each consonant and vowel is considered a token. ${ }^{3}$

\subsection{Tokenizer}

The meta-level tokens come into shape with the help of tokenizers in some cases. We implement seven tokenizers on the morpheme level Kkma, Hannanum, Komoran, Okt and MeCab from KoNLPy (Park and Cho, 2014), Kiwi (Korean Intelligent Word Identifier) ${ }^{4}$ and machine-learningbased Khaiii (Kakao Hangul Analyzer III) $)^{5}$ and two tokenizers -SentencePiece (SPM) (Kudo and Richardson, 2018) and $\mathrm{Jamo}^{6}$ - on the subword level.

\subsubsection{Tag Set}

Most Korean morphological analyzers have their roots in the 21st Century Sejong Project launched in 1998 intending to build a national framework for large-scale Korean corpora (21st Sejong Project, 1999). The tokenizers feature a different number of tag sets derived from the Sejong tag sets, as described in Appendix A.

The prototype tag set is preserved in Komoran and similarly in MeCab and Khaiii. The tokenizer with the most diminutive tag set is Kkma with 56 tags. It can provide a detailed analysis of endings (Eomi). The reverse case is Okt, a tokenizer targeted for Twitter, with 19 tags. Woo and Jung

\footnotetext{
${ }^{3}$ Although SPM is considered as a subword tokenizer in general, such a concept does not coincide with Korean linguistics. Hence, while its output takes the shape of morpheme, we categorize SentencePiece in the subword level.

${ }^{4}$ https: //github.com/bab2min/Kiwi

${ }^{5}$ https://github.com/kakao/khaiii

${ }^{6}$ https: //github.com/JDongian/ python-jamo
}

(2019) report its outstanding performance with typos, emojis, and punctuations. Hannanum also features a small-sized tag set (22 tags). Its exceptionally compressed division of particle tags is noticeable, among others. As expected, the central divergence of the tag sets is observed in particles, endings, and affixes.

\subsubsection{Tokenization Scenario}

As displayed in Table 2, the exemplary sentence is extracted from our data set to show a various tokenization scenario. It accumulates all possible scenarios of the meta-levels studied in our experiment.

The instance shows that the segmentation is most diversified in verbs (활보했다) with nine possibilities. It is also intriguing that some tokenizers consider Jongseong as an independent token (하, - ᄂ ). Such case is Hannanum, Kkma, Komoran, Khaiii and Kiwi.

\section{Experiment}

\subsection{Experiment Setup}

As Korean evaluation data is rarely available, we organized a human evaluation of four commercial NMT systems for the English-to-Korean translation direction with Direct Assessment (DA), the conventional human metric at Conference on Machine Translation (Barrault et al., 2020). Consequently, a machine evaluation with BLEU, TER, and ChrF of SacreBLEU is performed. With the resources at hand, the correlation between the two evaluation results is computed at the segment and corpus level.

\subsubsection{Dataset}

- Source Test Set: The original English texts are borrowed from WMT 2020 English IIItype test set, composed of 2,048 sentences (61 documents) with segment split. The segmentsplit format allows them the freedom of translating beyond a sentence level (Barrault et al., 2020). 
- Reference Translation: The Korean reference translation is created by a group of professional translators. The translators are advised not to post-edit MT. To guarantee the highest translation quality, we double-check the final revision. The revision is implemented only if the sentence is semantically erroneous.

- System Translation: We employ four online MT models including our own - Kakao $i^{7}$. They are denominated as $\operatorname{Sys}_{A}, \operatorname{Sys}_{B}, \operatorname{Sys}_{P}$ and $\operatorname{Sys}_{Q}$ in order to keep their anonymity for legal reason. The system translations are obtained on July 21, 2021.

In terms of normalizing data, errors in the source test sets and their subsequent impact on the system translations (Kim et al., 2021) remain undealt with. Only some minor technical issues, i.e. different single quotations (' and '), are normalized.

\subsubsection{Human Evaluation}

DA is a metric where an evaluator scores each sentence on a continuous scale of 0-100 in the category of Adequacy and Fluency. We hire 25 professional translators and assign each person a HIT of more or less 300 translated sentences with the context of the documents maintained. They are advised to consider the context when making a judgment. They are allowed to reverse their previous decisions within a document.

They are either holders of a master's degree in interpretation and translation in the English-Korean language pair or freelance translators with a minimum of two years of experience. In light of the fact that all participants are new to MT evaluation, we provide a detailed guideline for the experiment.

One judgment per system translation is gathered, amounting to 16,384 (8,192 of Adequacy and Fluency) evaluation data. The judgment on Fluency is only utilized as supplementary information.

\subsubsection{Quality Control}

Out of the 8,192 Adequacy judgments, the first ten judgments of each evaluator are considered an adjusting step, and so, they are removed. The scores are then normalized with judge-wise Zscores. With them, Inter-Quartile Range (IQR) is computed as in Equation 1, where $Q_{1}$ and $Q_{3}$ signify the first and third quartile values. The outlier $x$ belongs to the range that meets the condition.

\footnotetext{
${ }^{7}$ https: // www.translate.kakao.com
}

Having removed $5.67 \%$ of the data, we base our observation on 7,727 judgments.

$$
x<Q_{1}-1.5 \cdot\left(Q_{3}-Q_{1}\right)
$$

or

$$
x>Q_{3}+1.5 \cdot\left(Q_{3}-Q_{1}\right)
$$

\subsubsection{Computation}

The hypothesis and reference translations are tokenized by the aforementioned 11 token units without applying any additional normalization. Consequently, the scores of the automatic metrics are computed, and their Pearson's correlation coefficient $r$ to the the human Adequacy judgment are measured as such:

$$
r=\frac{\sum_{i=1}^{n}\left(H_{i}-\bar{H}\right) \cdot\left(M_{i}-\bar{M}\right)}{\sqrt{\sum_{i=1}^{n}\left(H_{i}-\bar{H}\right)^{2}} \sqrt{\sum_{i=1}^{n}\left(M_{i}-\bar{M}\right)^{2}}}
$$

$H$ and $M$ refer to the machine and human DA score, respectively, and $\bar{H}$ and $\bar{M}$, their mean values. The Pearson's $r$ measures the linear relationship between them.

During the process, some of the issues have concerned us:

\section{- Do we adjust n-gram parameters?}

By default, the BLEU score is a geometric mean of four-grams. As the token unit is divergent, on the one hand, we attempt to avoid a circumstance where any tokenizer benefits from the n-gram parameter. On the other hand, the default n-grams of $\mathrm{ChrF}$ at the word level are zero. To make the consequence of the token unit visible and compatible, we acquire in advance the best-correlated $n$-grams of each token unit to the human judgment. The ngrams of the token unit, therefore, is specified together with the outcome.

\section{- TER scores over 1.0?}

Theoretically speaking, TER $=1.0$ represents a perfect match between reference and hypothesis sentences. However, we detect that when a hypothesis is too short for its reference, the (sentence-level) TER score exceeds 1.0. Such cases are avoided by being normalized to 1.0 afterward.

\section{- Is there a tie rank?}

We run a Wilcoxon ranksum test to identify 


\begin{tabular}{|c|c|c|c|c|c|c|c|c|c|c|c|c|c|c|c|c|c|c|c|c|c|c|c|c|}
\hline & \multicolumn{8}{|c|}{ BLEU } & \multicolumn{8}{|c|}{ TER } & \multicolumn{8}{|c|}{$\mathrm{ChrF}$} \\
\hline & 500 & 1000 & 1500 & 2000 & 2500 & 3000 & 4000 & 5000 & 500 & 1000 & 1500 & 2000 & 2500 & 3000 & 4000 & 5000 & 500 & 1000 & 1500 & 2000 & 2500 & 3000 & 4000 & 5000 \\
\hline moran & 0.623 & 1.776 & 0.399 & & & & & & & & & & & & & & & & & & & & & 1.214 \\
\hline Chaiii & 0.392 & 1.985 & 0.426 & & & 0.978 & 0.058 & & & & & & & & & & & & & & & & & 1.262 \\
\hline iwi & & 1.868 & & & & 0.267 & & & & & & & & 4.039 & 3.165 & & 1.119 & 0.066 & 322 & 0.149 & .561 & & & 0.484 \\
\hline kma & & 1. & & & & 1.8 & & & & & & & & 4. & 4.666 & & & 0.815 & 195 & & 493 & 499 & 1.0 & 3.443 \\
\hline & & & & & & & & & & & & & & & & & & & & & & 1.776 & & 0.656 \\
\hline $\mathrm{Cab}$ & & & & & & 0.529 & 1.2 & & & & & & & & 4.5 & & & 0.5 & 0.603 & & 0.65 & 2.648 & 1.4 & 1.358 \\
\hline & & 0.957 & 1.5 & & & 2.052 & 1.6 & 1.7 & & 3 & & & 3.44 & & & & 0.5 & & 0.832 & 1.0 & 0.612 & 3.496 & $\begin{array}{l}1.659 \\
\text { S }\end{array}$ & 1.753 \\
\hline & & & 0.3 & & & & & & & & & & & & & & & & & & & & & $4.714^{4}$ \\
\hline & 1.8 & 2 & 3.4 & 1.2 & 3.6 & & 3.4 & 3.9 & & & & & & & & & & & & & & & & 1.2 \\
\hline nnan & 2.221 & 4.063 & 3.736 & 4.096 & $5.474 *$ & 4.507 & 4.691 & & & & & 0.9 & 1.6 & 0.69 & & 0.0 & 0.77 & $1.665^{*}$ & $1.934^{*}$ & & 1.3 & $4.212^{*}$ & $5.342^{*}$ & \\
\hline onc & 4.24* & $4.43^{*}$ & $5.124^{*}$ & $5.412^{*}$ & 5.078 & $5.93 *$ & $5.65^{*}$ & $5.984 *$ & $0.164 *$ & $0.062^{*}$ & $0.173^{*}$ & 0.629 & $0.492 *$ & $0.213^{*}$ & 0.663 & $0.041^{*}$ & 0.311 & 1.016 & 1.787 & 1.483 & 2.927 & 2.344 & 3.659 & 2.585 \\
\hline
\end{tabular}

Table 3: Average segment-wise ranks of the token unit for SacreBLEU resampled by $n$ samples. The highest ranking is in bold while the lowest is marked with asterisk (*).

\begin{tabular}{|c|c|c|c|c|c|c|c|c|}
\hline \multicolumn{3}{|c|}{ BLEU } & \multicolumn{2}{|c|}{ TER } & \multicolumn{4}{|c|}{ ChrF } \\
\hline & $r$ & $n$ & & $r$ & & $r$ & char $\_n$ & word_n $n$ \\
\hline Jamo & 0.3079 & 5 & Kkma & 0.2949 & Komoran & 0.3304 & 3 & 1 \\
\hline Kiwi & 0.3073 & 2 & Kiwi & 0.2933 & Khaiii & 0.3301 & 3 & 1 \\
\hline Komoran & 0.3062 & 2 & Mccab & 0.2912 & Kiwi & 0.3295 & 3 & 1 \\
\hline Character & 0.3054 & 2 & Komoran & 0.2898 & Kkma & 0.3263 & 3 & 0 \\
\hline Khaiii & 0.3051 & 2 & $\mathrm{Spm}$ & 0.2894 & Mecab & 0.3230 & 3 & 1 \\
\hline Kkma & 0.3019 & 2 & Khaiii & 0.2884 & Character & 0.3230 & 3 & 2 \\
\hline Mecab & 0.3017 & 2 & Character & 0.2860 & Spm & 0.3222 & 3 & 1 \\
\hline $\mathrm{Spm}$ & 0.3008 & 2 & Okt & 0.2776 & okt & 0.3208 & 3 & 1 \\
\hline Okt & 0.2843 & 2 & Jamo & 0.2765 & Hannanum & 0.3200 & 3 & 0 \\
\hline Hannanum & 0.2663 & 2 & Hannanum & 0.2503 & Word & 0.3199 & 3 & 0 \\
\hline Word & 0.2498 & 1 & Word & 0.2383 & Jamo & 0.3160 & 5 & 0 \\
\hline
\end{tabular}

Figure 1: Result of Pearson correlation $r$ with ranking clusters by Wilcoxon ranksum test at the segment level. The token units in the identical background color are considered tie. The adjustment of n-grams is specified in the $n$ columns.

the statistical significance between the ranks. First, we set a cluster boundary with the pvalue based on the assumption that two token types whose $\mathrm{p}$-value is less than $0.05(p<$ 0.05 ) are statistically different. After counting such cases for all combinations, those with the same number of counts are considered a tie.

\section{- Is the sample size enough?}

To yield a credible result, we apply Bootstrap Resampling suggested by Koehn (2004). Out of 7,727 sentences, different blocks of subsamples are extracted in a binary round ( $N$ out of 7,727 and $M$ out of $N$ ) on a random basis. Iterating $I$ times, $M$ out of $M$ samples are randomly selected to print the final result. Koehn (2004) reported that they reached a $95 \%$ confidence level with 394 samples $(N)$ and near $100 \%$ with 3,000 samples when assessing MT systems with BLEU. While we follow the precedence by setting up the parameters similarly as $M=6000, N=3000, I=1000$, we provide additional results with variations in $N$ and $I$ in Table 3.

\subsection{Experiment Result}

BLEU, TER, and ChrF scores and their correlation results are analyzed at the segment/corpus level. While finding the best pre-tokenization scheme is intriguing, our primary focus is to examine that SacreBLEU results are susceptible to the pretokenization scenarios.

\subsubsection{Segment Level}

Table 1 shows the Pearson correlation of the token units clustered by Wilcoxon ranksum test. The result of Bootstrap Resampling is given in Table 3.

BLEU. Without resampling, the result shows that the highest correlation is observed in a broad spectrum of token units: Jamo, Kiwi, Komoran and Character. The lowest correlation is when pre-tokenization is absent, which is consistently witnessed in the resampled outcome, except for one instance of Hannanum $(n=2500)$. It is safe to conclude, therefore, that any tokenizer can enhance the credibility of this metric, but Hannanum is not an option. Besides, we also report the moderate impact of MeCab.

TER. Before resampling, the morpheme level (Kiwi and Kkma) goes best with this metric, followed by MeCab. Considering the lowest correlation of the word level, it is a reasonable guess that pre-tokenization is essential in this metric. Such trend is still valid when the data is resampled, aside from the two negative cases in Jamo. Among the tokenizers, the positive influence of Kkma is noteworthy as the sample size grows. Moreover, MeCab seems to be a good fit for this metric in Korean. The least recommendable token unit is Jamo, not only because its correlation is low on average but also the increased token size is markedly disadvantageous to the computational cost of this metric. 
ChrF. With the 7,727 data set, the optimal token unit for this metric is morpheme (Komoran and Khaiii). Komoran is remarkably well-fitted to this metric even when the data is resampled. However, the most differential aspect of this metric is that tokenization can be harmful. For instance, in a pilot study we find that the best-correlated word n-grams for Kkma and Hannanum are zero, meaning that it is best when they are not taken into consideration. All but the character level have a history of deteriorating the correlation of the metric. In that respect, Hannanum and Jamo produce the most unstable result. It draws our attention that when the sample size is small $(n=500)$, most of the pre-tokenization worsens its correlation, and $\mathrm{MeCab}$ is not an exception.

All in all, we conclude that at the segment level, the correlation of the three metrics fluctuates by the pre-tokenization scheme, but there is no direct relationship between the token shape and the correlation of the metrics. Nevertheless, if we are to draw a meaningful conclusion from the result, any pre-tokenization is better than the word level in BLEU and TER, but in ChrF the token unit should be carefully selected. The combination of $\mathrm{MeCab}$ and BLEU/TER stands out, while the popular tokenizer can detriment the credibility of ChrF.

Unlike our expectations, the subword level does not serve as a dependable token unit for the Korean MT evaluation. Instead, there is a good chance that it harms the correlation of ChrF and TER. Furthermore, the expanded vocab size increases the computational cost exponentially. As a substitute, we highlight the effectiveness of the character-level segmentation, which guarantees a fast deployment without any tokenizer and, at the same time, is proven to be as reliable as or often better than $\mathrm{MeCab}$ in all metrics.

\subsubsection{Corpus Level}

Table 4 - 6 show human DA scores and SacreBLEU scores of four online systems in relation to the tokenization scheme. The noticeable finding is that in all three cases the spectrum of the score is substantial. The highest-ranked system in the human z-score $\left(S y s_{A}\right)$ obtained a 28.09 BLEU score without segmentation, but 48.71 when with the character-level tokenization. Likewise, the most overestimated version of TER is before tokenization (82.33 - 89.69), as expected, while the most underestimated version of score is on the Jamo level
(51.96 - 54.69). In ChrF, the range mentioned above is from $42.74-45.72$ on the None level to 51.19 53.80 on the Jamo level. We, thus, confirm the possibility that the absolute score of SacreBLEU can be doubled just by selecting a different tokenizer.

While so, the more severe problem is that the ranks by score, irrelevant from the pre-tokenization typology, do not comply with human perception. The human average scores place the systems in the order of $\left[\right.$ Sys $_{A}=1$, Sys $_{B}=2$, Sys $_{P}=$ $\left.3, S y s_{Q}=4\right]$, but almost all automatic scores position them as $\left[\right.$ Sys $_{A}=2$, Sys $_{B}=1$, Sys $_{P}=$ $\left.3, S y s_{Q}=4\right]$. Moreover, in many cases the BLEU scores are prone to rank them as $\left[\right.$ Sys $_{A}=$ 2, Sys $_{B}=1$, Sys $_{P}=4$, Sys $\left._{Q}=3\right]$ except when tokenized by MeCab, Kiwi and Khaiii. Such an unreliable performance of this metric can be derived from either the small number of systems or the existence of outlier systems in the comparison (Mathur et al., 2020). We raise the issue of a questionable SacreBLEU score at the corpus level, leaving its verification to our future work.

Figure 2 shows the Pearson correlation of SacreBLEU when with the pre-tokenization. The most faithful score is achieved with Khaiii and Kiwi in all three metrics. The correlation of $\mathrm{MeCab}$ is also striking. Despite their discernible performance on this level, however, we reiterate that none of the options represent the system rankings as humans perceive. In this respect, we propose the mean value of segment-wise SacreBLEU score as a substitute.

\section{Related Works}

Recently, a word segmentation got the limelight with the outstanding achievement of subword-level pipelines such as SPM or Byte-Pair Encoding (BPE) (Sennrich et al., 2015) in many NLP tasks (Zhang et al. 2015; ). In MT, in specific, the segmentation is tightly entangled with the translation quality mainly due to the handling of unseen vocabulary. In that respect, many studies observed that identifying the best tokenization is languagedependant.

Huck et al. (2017) discovered that their model displayed the highest performance when BPE was coupled with a suffix split in German. In a similar manner, Lee et al. (2016) suggested that their fully character-level NMT model outperformed BPE models, especially in the Finnish-English pair. Domingo et al. (2018) demonstrated that when five languages were under study, no single best tok- 


\begin{tabular}{llllllllllllll}
\hline & Ave. $\uparrow$ & Ave. z & None & Okt & Mecab & Komoran & Kkma & Kiwi & Khaiii & Hannanum & Character & Spm & Jamo \\
\hline Sys $_{A}$ & $\mathbf{6 8 . 7 8 3}$ & $\mathbf{0 . 2 0 3}$ & 28.099 & 33.398 & 38.341 & 40.275 & 40.986 & 41.022 & 40.005 & 36.939 & 48.712 & 41.015 & 48.467 \\
Sys $_{B}$ & 67.160 & 0.112 & $\mathbf{2 8 . 9 3 2}$ & $\mathbf{3 4 . 3 5 1}$ & $\mathbf{3 9 . 1 8 5}$ & $\mathbf{4 1 . 0 0 7}$ & $\mathbf{4 1 . 9 2 0}$ & $\mathbf{4 1 . 9 9 7}$ & $\mathbf{4 0 . 8 8 1}$ & $\mathbf{3 7 . 7 9 3}$ & $\mathbf{4 9 . 5 5 3}$ & $\mathbf{4 1 . 9 4 8}$ & $\mathbf{4 9 . 1 8 8}$ \\
Sys $_{P}$ & 64.688 & 0.027 & 23.941 & 30.415 & 35.605 & 36.621 & 37.236 & 38.458 & 37.034 & 32.902 & 45.924 & 37.213 & 45.098 \\
Sys $_{Q}$ & 57.734 & -0.220 & 25.941 & 31.382 & 35.602 & 37.304 & 38.063 & 38.138 & 36.939 & 34.058 & 47.096 & 38.155 & 46.602 \\
\hline
\end{tabular}

Table 4: The variation of BLEU score of the four MT systems by token type along with human DA scores and their $\mathrm{z}$-scores. The best scores are in bold.

\begin{tabular}{|c|c|c|c|c|c|c|c|c|c|c|c|c|c|}
\hline & Ave. $\uparrow$ & Ave. $z$ & None & Okt & Mecab & Komoran & Kkma & Kiwi & Khaiii & Hannanum & Character & Spm & Jamo \\
\hline$S y s_{A}$ & 68.783 & 0.203 & 82.811 & 68.223 & 64.142 & 63.041 & 62.253 & 62.352 & 63.412 & 67.833 & 57.718 & 62.391 & 52.932 \\
\hline$S y s_{B}$ & 67.160 & 0.112 & 82.334 & 67.332 & 63.519 & 62.585 & 61.545 & 61.649 & 62.867 & 67.249 & 56.364 & 61.083 & 51.962 \\
\hline Sys $_{P}$ & 64.688 & 0.027 & 89.652 & 69.882 & 64.898 & 64.859 & 63.479 & 62.983 & 64.346 & 71.199 & 62.163 & 65.914 & 54.063 \\
\hline$S y s_{Q}$ & 57.734 & -0.220 & 86.699 & 70.356 & 66.611 & 65.641 & 64.751 & 64.758 & 66.126 & 71.199 & 59.771 & 64.767 & 54.697 \\
\hline
\end{tabular}

Table 5: The variation of TER score of the four MT systems by token type along with human DA scores and their z-scores. The best scores are in bold.

\begin{tabular}{|c|c|c|c|c|c|c|c|c|c|c|c|c|c|}
\hline & Ave. $\uparrow$ & Ave. $z$ & None & Okt & Mecab & Komoran & Kkma & Kiwi & Khaiii & Hannanum & Character & Spm & Jamo \\
\hline Sys $_{A}$ & 68.783 & 0.203 & 44.897 & 46.508 & 47.544 & 48.904 & 46.326 & 49.299 & 48.763 & 46.019 & 47.887 & 47.932 & 53.140 \\
\hline $\operatorname{Sys}_{B}$ & 67.160 & 0.112 & 45.725 & 47.345 & 48.370 & 49.635 & 47.131 & 50.096 & 49.560 & 46.826 & 48.707 & 48.807 & 53.807 \\
\hline Sys $_{P}$ & 64.688 & 0.027 & 42.742 & 44.171 & 45.342 & 46.182 & 43.796 & 47.017 & 46.354 & 43.401 & 45.699 & 45.357 & 51.198 \\
\hline $\operatorname{Sys}_{Q}$ & 57.734 & -0.220 & 43.505 & 45.134 & 46.031 & 47.166 & 44.639 & 47.557 & 47.011 & 44.378 & 46.533 & 44.378 & 51.775 \\
\hline
\end{tabular}

Table 6: The variation of ChrF score of the four MT systems by token type along with human DA scores and their z-scores. The best scores are in bold.
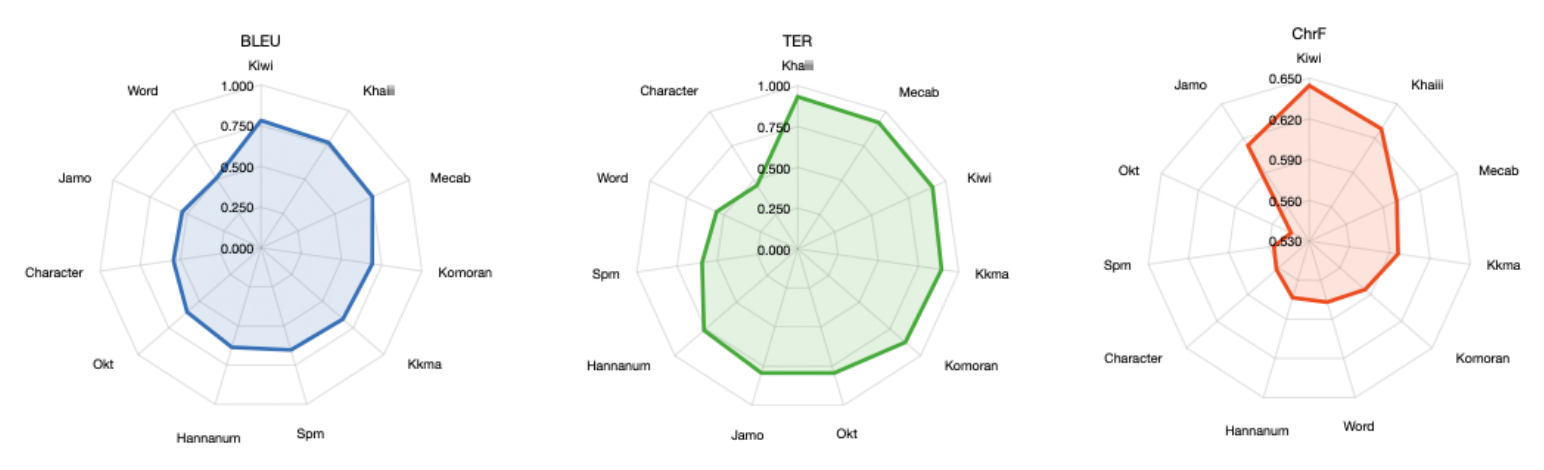

Figure 2: Result of Pearson correlation $r$ at the corpus level. 
enizer could lead to a more refined translation quality for all languages. They made a remark that such phenomenon was striking in morphologically rich languages such as Japanese.

In relation to Korean in this regard, the concept of detailed segmentation intrigued many researchers in NLP in general (Park et al. 2018; Kim et al. 2020; Yongseok and Lee 2020; Park et al. 2020), for a Korean word had a large volume of affixes and morphemes. In MT in specific, various combinations of token units were suggested. Among them, Park et al. (2019) stated that when their NMT model was trained with a dataset of subtitles tokenized with SPM Unigram after removing postpositional particles, it obtained a higher BLEU score than when with simple BPE.

While they mentioned that smaller token unit was not always an answer in the case of Korean, recent studies paid attention to a smallest unit, the Jamo. Moon and Okazaki (2020) introduced JamoPair Encoding, combining Jamo with BPE. Eo et al. (2021) utilized Jamo from a functional viewpoint by regarding Choseong and Jungseong as one token and leaving Jongseong as another. They demonstrated that the model with such segmentation outperformed the model of Park et al. (2019).

We differ from the aforementioned studies in that we explore the impact of tokenization on the MT evaluation. As the gold standard in this field is human, we prioritize the examination of the pattern of its impact on SacreBLEU, instead of discovering a superior token unit. Assuming that the matching system of $\mathrm{N}$-grams in BLEU and $\mathrm{ChrF}$ or edit distance in TER is exceptionally vulnerable to the lexical shape of tokens, we observe how such changes are in line with human perception. We believe it is a pitfall to the MT evaluation of synthetic languages ${ }^{8}$ such as Korean.

\section{Conclusion}

On the condition that pre-tokenization is obligatory for the agglutinative language such as Korean when computing automatic evaluation metrics, we endeavor to demonstrate the influence of dynamic token units on the credibility of SacreBLEU, including BLEU, TER, and ChrF at both the segment and corpus level in the into-Korean translation direction.

For the meta-evaluation, we perform a human

\footnotetext{
${ }^{8}$ It refers to a language group whose word is composed of an exceptional number of morphemes.
}

evaluation with 25 professional translators on system translations produced by four NMT models. When the Pearson correlation is measured, the result shows that the impact of token type differs from metric and its computation level. At the segment level, we report that any pre-tokenization enhances the correlation of BLEU and TER while it should be carefully selected in ChrF. At the corpus level, ranking by the SacreBLEU scores turns out to be inaccurate regardless of the pre-tokenization scheme.

Contrary to our expectation, the diminutive segmentation by the subword level shows signs of ineffectiveness. Instead, we put an emphasis on the role of the character level.

\section{Acknowledgements}

Special thanks to our team members for their thoughtful comments and healthy discussions.

\section{References}

The 21st Sejong Project. 1999. Construction of korean basic data (academic service report).

Loïc Barrault, Magdalena Biesialska, Ondřej Bojar, Marta R. Costa-jussà, Christian Federmann, Yvette Graham, Roman Grundkiewicz, Barry Haddow, Matthias Huck, Eric Joanis, Tom Kocmi, Philipp Koehn, Chi-kiu Lo, Nikola Ljubešić, Christof Monz, Makoto Morishita, Masaaki Nagata, Toshiaki Nakazawa, Santanu Pal, Matt Post, and Marcos Zampieri. 2020. Findings of the 2020 conference on machine translation (WMT20). In Proceedings of the Fifth Conference on Machine Translation, pages 1-55, Online. Association for Computational Linguistics.

Chris Callison-Burch, Miles Osborne, and Philipp Koehn. 2006. Re-evaluating the role of Bleu in machine translation research. In 11th Conference of the European Chapter of the Association for Computational Linguistics, pages 249-256, Trento, Italy. Association for Computational Linguistics.

Miguel Domingo, Mercedes García-Martínez, Alexandre Helle, Francisco Casacuberta, and Manuel Herranz. 2018. How much does tokenization affect neural machine translation? CoRR, abs/1812.08621.

Sugyeong Eo, Chanjun Park, Hyeonseok Moon, and Heuiseok Lim. 2021. Research on subword tokenization of korean neural machine translation and proposal for tokenization method to separate jongsung from syllables. Journal of the Korea Convergence Society, 12(3):1-7.

Matthias Huck, Simon Riess, and Alexander M. Fraser. 2017. Target-side word segmentation strategies for neural machine translation. In WMT. 
Ahrii Kim, Yunju Bak, Jimin Sun, Sungwon Lyu, and Changmin Lee. 2021. The suboptimal wmt test sets and their impact on human parity. Preprints.

Hwichan Kim, Tosho Hirasawa, and Mamoru Komachi. 2020. Zero-shot North Korean to English neural machine translation by character tokenization and phoneme decomposition. In Proceedings of the 58th Annual Meeting of the Association for Computational Linguistics: Student Research Workshop, pages 72 78, Online. Association for Computational Linguistics.

Philipp Koehn. 2004. Statistical significance tests for machine translation evaluation. In Proceedings of EMNLP 2004, pages 388-395. Association for Computational Linguistics.

Taku Kudo and John Richardson. 2018. SentencePiece: A simple and language independent subword tokenizer and detokenizer for neural text processing. In Proceedings of the 2018 Conference on Empirical Methods in Natural Language Processing: System Demonstrations, pages 66-71, Brussels, Belgium. Association for Computational Linguistics.

Jason Lee, Kyunghyun Cho, and Thomas Hofmann. 2016. Fully character-level neural machine translation without explicit segmentation. CoRR, abs/1610.03017.

Benjamin Marie, Atsushi Fujita, and Raphael Rubino. 2021. Scientific credibility of machine translation research: A meta-evaluation of 769 papers. CoRR, abs/2106.15195.

Nitika Mathur, Tim Baldwin, and Trevor Cohn. 2020 Tangled up in BLEU: reevaluating the evaluation of automatic machine translation evaluation metrics. CoRR, abs/2006.06264.

Sangwhan Moon and Naoaki Okazaki. 2020. Jamo pair encoding: Subcharacter representation-based extreme Korean vocabulary compression for efficient subword tokenization. In Proceedings of the 12th Language Resources and Evaluation Conference, pages 3490-3497, Marseille, France. European Language Resources Association.

Toshiaki Nakazawa, Hideki Nakayama, Chenchen Ding, Raj Dabre, Shohei Higashiyama, Hideya Mino, Isao Goto, Win Pa Pa, Anoop Kunchukuttan, Shantipriya Parida, Ondřej Bojar, and Sadao Kurohashi. 2020. Overview of the 7th workshop on Asian translation. In Proceedings of the 7th Workshop on Asian Translation, pages 1-44, Suzhou, China. Association for Computational Linguistics.

Gisim Nam, Yeonggeun Ko, Hyunkyung Yu, and Hyeongyong Choi. 2019. Korean standard grammar (표준 국어문법론). Hankook Munhwasa, Korea.

Kishore Papineni, Salim Roukos, Todd Ward, and WeiJing Zhu. 2002. Bleu: A method for automatic evaluation of machine translation. In Proceedings of the 40th Annual Meeting on Association for Computational Linguistics, ACL '02, page 311-318, USA. Association for Computational Linguistics.

Chanjun Park, Gyeongmin Kim, and Heuiseok Lim. 2019. Parallel corpus filtering and korean-optimized subword tokenization for machine translation. Annual Conference on Human and Language Technology, pages 221-224.

Eunjeong L. Park and Sungzoon Cho. 2014. Konlpy: Korean natural language processing in python. In Proceedings of the 26th Annual Conference on $\mathrm{Hu}$ man Cognitive Language Technology, Chuncheon, Korea.

Kyubyong Park, Joohong Lee, Seongbo Jang, and Dawoon Jung. 2020. An empirical study of tokenization strategies for various korean NLP tasks. CoRR, abs/2010.02534.

Sungjoon Park, Jeongmin Byun, Sion Baek, Yongseok Cho, and Alice Oh. 2018. Subword-level word vector representations for Korean. In Proceedings of the 56th Annual Meeting of the Association for Computational Linguistics (Volume 1: Long Papers), pages 2429-2438, Melbourne, Australia. Association for Computational Linguistics.

Maja Popović. 2015. chrF: character n-gram F-score for automatic MT evaluation. In Proceedings of the Tenth Workshop on Statistical Machine Translation, pages 392-395, Lisbon, Portugal. Association for Computational Linguistics.

Matt Post. 2018. A call for clarity in reporting BLEU scores. CoRR, abs/1804.08771.

Rico Sennrich, Barry Haddow, and Alexandra Birch. 2015. Neural machine translation of rare words with subword units. CoRR, abs/1508.07909.

Matthew Snover, Bonnie Dorr, Richard Schwartz, Linnea Micciulla, and John Makhoul. 2006. A study of translation edit rate with targeted human annotation. In In Proceedings of Association for Machine Translation in the Americas, pages 223-231.

Liling Tan, Jon Dehdari, and Josef van Genabith. 2015. An awkward disparity between BLEU / RIBES scores and human judgements in machine translation. In Proceedings of the 2nd Workshop on Asian Translation (WAT2015), pages 74-81, Kyoto, Japan. Workshop on Asian Translation.

Kyungjin Woo and Suhyeon Jung. 2019. Comparison of korean morphology analyzers according to the types of sentence. Proceedings of the Korean Information Science Society Conference, pages 1388-1390.

Choi Yongseok and Kongjoo Lee. 2020. Performance analysis of korean morphological analyzer based on transformer and bert. Journal of KIISE, 47(8):730741.

Xiang Zhang, Junbo Jake Zhao, and Yann LeCun. 2015. Character-level convolutional networks for text classification. CoRR, abs/1509.01626. 


\section{A Appendix}

\begin{tabular}{|c|c|c|c|c|c|c|c|c|c|c|}
\hline & Category & & Sejong & Okt & Komoran & MeCab-ko & Kkma & Hannanum & Khaiii & Kiwi \\
\hline & \# of tags & & 42 & 19 & 42 & 43 & 56 & 22 & 46 & 47 \\
\hline \multirow{6}{*}{ Substantive } & \multirow{4}{*}{ noun } & general & NNG & \multirow{6}{*}{ Noun } & NNG & NNG & NNG & $\mathrm{NC}$ & NNG & NNG \\
\hline & & proper & NNP & & NNP & NNP & NNP & NQ & NNP & NNP \\
\hline & & dependent & \multirow[t]{2}{*}{ NNB } & & NNB & NNB & NNB & NB & NNB & NNB \\
\hline & & unit & & & NNDD & NNBC & NNM & ND & NIVD & NVID \\
\hline & \multicolumn{2}{|c|}{ pronoun } & NP & & NP & NP & NP & NP & $\mathrm{NP}$ & NP \\
\hline & \multicolumn{2}{|c|}{ numeral } & NR & & NR & NR & NR & NN & NR & NR \\
\hline \multirow{5}{*}{ Predicate } & \multicolumn{2}{|c|}{ verb } & VV & Verb & VV & VV & VV & PV & VV & VV \\
\hline & adjective & & VA & Adjective & VA & VA & VA & PA & VA & VA \\
\hline & \multicolumn{2}{|c|}{ auxiliary } & VX & - & vX & vX & $\begin{array}{l}\text { VXV } \\
\text { VXA }\end{array}$ & PX & VX & $\mathrm{vX}$ \\
\hline & \multirow{2}{*}{ copula } & positive & $\mathrm{VCP}$ & - & VCP & $\mathrm{VCP}$ & VCP & - & VCP & $\mathrm{VCP}$ \\
\hline & & negative & VCN & - & VCN & VCN & VCN & - & VCN & VCN \\
\hline \multirow{3}{*}{ Modifier } & article & $\begin{array}{c}\text { determiner } \\
\text { numeral }\end{array}$ & MM & Determiner & MM & MM & $\begin{array}{l}\text { MDT } \\
\text { MDN }\end{array}$ & MM & MM & MM \\
\hline & dverb & general & MAG & Adverb & MAG & MAG & MAG & $M$ & MAG & MAG \\
\hline & adverb & connective & MAJ & Conjunction & MAJ & MAJ & MAC & MA & MAJ & MAJ \\
\hline Interjection & interjectic & & IC & Exclamation & IC & IC & IC & II & IC & IC \\
\hline & & subjective & JKS & & JKS & JKS & JKS & & JKS & JKS \\
\hline & & complement & JKC & & JKC & JKC & JKC & & JKC & JKC \\
\hline & & adnominal & JKG & & JKG & JKG & JKG & & JKG & JKG \\
\hline & case-marking & objective & JKO & & JKO & JKO & JKO & $\mathrm{JC}$ & JKO & JKO \\
\hline Dostnocitional Particl & & adverbial & JKB & & JKB & JKB & JKM & & JKM & JKM \\
\hline Post-positional Particle & & vocative & JKV & Josa & JKV & JKV & JKI & & JKI & JKI \\
\hline & & quotation & JKQ & & JKQ & JKQ & JKQ & & JKQ & JKQ \\
\hline & auxiliary & & $\mathrm{JX}$ & & $\mathrm{JX}$ & $\mathrm{JX}$ & $\mathrm{JX}$ & IX & $\mathrm{JX}$ & $\mathrm{JX}$ \\
\hline & conjuncti & & $\mathrm{JC}$ & & $\mathrm{JC}$ & $\mathrm{JC}$ & $\mathrm{JC}$ & $J_{X}$ & $\mathrm{JC}$ & $\mathrm{JC}$ \\
\hline & predicati & & - & & - & - & - & JP & - & - \\
\hline & & honorific & & & & & EPH & & & \\
\hline & pre-final ending & tense & EP & PreEomi & EP & EP & EPT & EP & EP & EP \\
\hline & & politeness & & & & & EPP & & & \\
\hline & & declarative & & & & & EFN & & & \\
\hline & & interrogative & & & & & EFQ & & & \\
\hline & sentence-closing onding & imperative & FF & Fomi & & $\mathrm{FE}$ & EFO & $\mathrm{FF}$ & FF & $\mathrm{FF}$ \\
\hline & sentence-closing ending & requesting & $\mathrm{EF}$ & Eoml & & $\mathrm{EF}$ & EFA & $\mathrm{EF}$ & $\mathrm{EF}$ & $\mathrm{EF}$ \\
\hline & & interjective & & & $\mathrm{EF}$ & & EFI & & & \\
\hline & & honorific & & & & & EFR & & & \\
\hline Denendent & & equal & & & & & ECE & & & \\
\hline Dependent & connectiveending & auxiliary & $\mathrm{EC}$ & & & EC & ECS & $\mathrm{EC}$ & $\mathrm{EC}$ & $\mathrm{EC}$ \\
\hline & & dependent & & EC & & & ECD & & & \\
\hline & transformative ending & nominal & ETN & & ETN & ETN & ETN & ET & ETN & ETN \\
\hline & transformative enaing & adnominal & ETM & & ETM & ETM & ETD & E1 & ETD & ETD \\
\hline & nrefix & substantive & XPN & - & XPN & XPN & XPN & $X P$ & XPN & XPN \\
\hline & prenx & predicative & - & - & - & - & XPV & $\mathrm{XP}$ & - & - \\
\hline & & derived noun & XSN & & XSN & XSN & $\mathrm{XSN}$ & & $\mathrm{XSN}$ & $\mathrm{XSN}$ \\
\hline & suffix & derived verb & XSV & Suffix & XSV & XSV & XSV & XS & XSV & XSV \\
\hline & & derived adverb & XSA & & XSA & XSA & XSA & & XSA & XSA \\
\hline & root & root & $\mathrm{XR}$ & - & $\mathrm{XR}$ & $\mathrm{XR}$ & $\mathrm{XR}$ & - & XR & $\mathrm{XR}$ \\
\hline &.$? !$ & & $\mathrm{SF}$ & Punctuation & $\mathrm{SF}$ & $\mathrm{SF}$ & $\mathrm{SF}$ & & SF & SF \\
\hline & $\ldots$ & & SE & & SE & SE & SE & & SE & SE \\
\hline Punctuation & $", ", '()$ & & SS & & SS & $\begin{array}{l}\text { SSO여는 괄호 (, [ } \\
\text { SSC닫는 괄호 ) }]\end{array}$ & SS & $\mathrm{S}$ & SS & SS \\
\hline & $\sim$ - $_{-}$ & & SP & & SP & SC & SP & & SP & SP \\
\hline & others & & SO & & SO & SY & SO & & SO & SO \\
\hline & Chinese char & acter & SW & & SW & & SW & & SW & SW \\
\hline & foreign wo & & SH & Foreign & SH & SH & $\mathrm{OH}$ & F & SH & SH \\
\hline & number & & SL & Alpha & SL & SL & OL & & SL & SL \\
\hline & unknown n & & SN & Number & SN & SN & ON & - & SN & SN \\
\hline & unknown v & & $\mathrm{NF}$ & & $\mathrm{NF}$ & - & & - & $\mathrm{ZN}$ & \\
\hline & unknowı & & NV & Unknown & NV & - & UN & - & $\mathrm{ZV}$ & UN \\
\hline Etc. & unknowi & & NA & & NA & - & & - & $\mathrm{ZZ}$ & \\
\hline & consonant/v & wel & - & KoreanParticle & - & - & - & - & SWK & - \\
\hline & hashtag & & - & Hashtag & - & - & - & - & - & W_HASHTAG \\
\hline & user nam & & - & ScreenName & - & - & - & - & - & W_MENTION \\
\hline & email & & - & Email & - & - & - & - & - & W_EMAIL \\
\hline & url & & - & URL & - & - & - & - & - & W_URL \\
\hline
\end{tabular}

Table 7: Summary of the tag sets of Sejong and seven tokenizers. The tag sets of KoNLPy tokenizers are referred to KoNLPy. 9 